\title{
Expression of intronic miRNAs and their host gene lgf2 in a murine unilateral ureteral obstruction model
}

\author{
N.Q. $\mathrm{Li}^{1}$, J. Yang ${ }^{2}$, L. Cui ${ }^{1}$, N. Ma ${ }^{3}$, L. Zhang ${ }^{1}$ and L.R. Hao ${ }^{1}$ \\ ${ }^{1}$ Nephrology Department, The First Affiliated Hospital, Harbin Medical University, Harbin, China \\ ${ }^{2}$ Nephrology Department, Daqing Oilfield General Hospital, Daqing, China \\ ${ }^{3}$ Department of Biochemistry and Molecular Biology, Harbin Medical University, Harbin, China
}

\begin{abstract}
The objective of this study was to determine the expression of miR-483 and miR-483* and the relationship among them, their host gene (Igf2), and other cytokines in a murine model of renal fibrosis. The extent of renal fibrosis was visualized using Masson staining, and fibrosis was scored 3 days and 1 and 2 weeks after unilateral ureteral obstruction (UUO). Expression of miR-483, miR-483* and various cytokine mRNAs was detected by real-time polymerase chain reaction (PCR). Expression of miR-483 and miR-483* was significantly upregulated in the UUO model, particularly miR-483 expression was the greatest 2 weeks after surgery. Additionally, miR-483 and miR-483* expression negatively correlated with $B m p 7$ expression and positively correlated with Igf2, Tgf $\beta$, Hgf, and Ctgf expression, as determined by Pearson's correlation analysis. Hgf expression significantly increased at 1 and 2 weeks after the surgery compared to the control group. This study showed that miR-483 and miR-483* expression was upregulated in a murine UUO model. These data suggest that miR-483 and miR-483* play a role in renal fibrosis and that miR-483* may interact with miR-483 in renal fibrosis. Thus, these miRNAs may play a role in the pathogenesis of renal fibrosis and coexpression of their host gene lgf2.
\end{abstract}

Key words: microRNA; miR-483; Renal fibrosis; Igf2, UUO

\section{Introduction}

Progressive renal fibrosis is the end stage of various chronic kidney diseases and results in impaired renal function and, ultimately, kidney failure. Renal fibrosis is characterized by tubular cell apoptosis, leukocyte infiltration, necrosis, tubulointerstitial fibroblast proliferation, increased extracellular matrix (ECM) production, and the replacement of normal renal tissue. Despite fundamental advances in the understanding of the pathophysiology of renal fibrosis, definitive therapies remain limited.

The key issues for pathogenesis of renal fibrosis are still unclear. Renal fibrosis obviously is not a solitary disease, but involves a complicated network of mechanisms. In recent years, research has identified that many factors participate in renal fibrosis, including enhanced expression of proapoptotic genes and profibrotic growth factors, increased macrophage infiltration, and initiation of epithelial mesenchymal transition (EMT), as well as endothelial mesenchymal transition. Transforming growth factor- $\beta 1$ is one of the most important mediators of fibrogenesis and also regulates macrophage recruitment in the kidneys $(1,2)$.
Recently, microRNA (miRNA) has attracted attention after being shown to participate in a variety of physiological processes and the pathogenesis of human diseases. miRNAs are small ( $\sim 22 \mathrm{nt}$ ) endogenous noncoding RNAs that posttranscriptionally repress gene expression, mainly by binding to the $3^{\prime}$-untranslated regions of their target messenger RNAs. Abnormal miRNA expression is linked to the initiation and progression of pathological processes, including diabetes, cancer, cardiovascular disease, and osteoarthritis (3-5).

Current studies have indicated that abnormal miRNA expression is related to the pathogenesis of renal fibrosis (e.g., miR-21, miR-200, and miR-192). However, whether their host genes play a role in renal fibrosis, like the miRNAs, has not been determined. For example, in osteoarthritis, the overexpression of miR-675 and its host gene $\mathrm{H} 19$ may support cartilage anabolism and enhance tissue regeneration (6). Thus, miRNAs and their host genes may be involved in physiological or pathological processes together. Birnbacher et al. (7) reported that Igf2

Correspondence: Lirong Hao: <haolirong2013@sohu.com>.

Received May 1, 2014. Accepted October 22, 2014. First published online March 27, 2015. 
mRNA is expressed in the interstitial cells in the medullary region of the kidney and is involved in kidney development. Whether Igf2 and its intronic miRNA play a role in renal fibrosis is still unknown.

Additionally, miR-483, a conserved sequence encoded by intron 2 of $\operatorname{lgf2}$, was identified. The premiRNA, miR-483, generates two mature miRNAs, miR483-5p and miR-483-3p. Research has shown that abnormal miR-483 expression is associated with cancers, such as adrenocortical cancer and rectal cancer $(8,9)$. Ma et al. (10) reported that miR-483-5p is coexpressed with its host gene Igf2 in murine hepatocellular carcinoma cell lines. Thus, miR-483-5p can be viewed as a functional partner for lgf2. However, whether miR-483 and lgf2 expression are both abnormal and whether miR-483 is associated with its host gene in renal fibrosis are unknown. Cytokines are known to participate in renal fibrosis. Additionally, miR-483 might participate in regulating the balance between promoters and inhibitors in renal fibrosis by regulating the expression of some cytokines, such as bone morphogenetic protein-7 (BMP7), transforming growth factor beta (TGF $\beta$ ), hepatocyte growthpromoting factor, and connective tissue growth factor (CTGF). Therefore, this study aimed to assess miR-483 and miR-483* expression and to determine the relationships among the expression of miR-483, miR-483*, Igf2, and other cytokines in a unilateral ureteral obstruction (UUO) mouse model of renal fibrosis.

\section{Material and Methods}

\section{Experimental renal fibrosis model}

Twenty mice (male, 4-5 months old) were randomly and evenly divided into 4 groups. The UUO model was established as previously described (11). The mice were anesthetized with $0.5 \%$ pentobarbital sodium and placed on a heating pad at $37^{\circ} \mathrm{C}$. Through a midline incision, the right ureter was identified, ligated with 4-0 sutures, and cut at 2 sites near the renal hilum. After surgery, the mice were killed at 3 days and 1 and 2 weeks after surgery ( $n=5 /$ group). Five mice that did not undergo surgery were killed and served as a control group. The right kidneys of the mice were harvested, and the upper third of the kidney was used for RNA extraction, the middle third for histopathological analysis, and the lower third for Western blot analysis. The study was performed in compliance with the regulations of the Harbin Medical University Ethics Council, and the protocol was approved by the Experimental Animal Care and Use Committee of Harbin Medical University.

\section{Histopathological and semiquantitative evaluation of fibrosis}

To investigate fibrosis in the UUO mice, their kidneys were harvested and fixed using $4 \%$ paraformaldehyde. Three-microns-thick paraffin sections were Masson stained, and two independent observers examined the slides by light microscopy (Olympus BX41, Japan) using a modified scoring system. Grades were assigned to each slide according to the following criteria: 0 , no fibrosis; 1 , mild fibrosis (10-30\% in area); 2 , moderate fibrosis (30$50 \%$ in area); 3 , severe fibrosis ( $>50 \%$ in area). The scoring system was based on the average extent of histological change observed on four slides.

\section{Western blot analysis}

Using a homogenate bar, the lower third of each kidney was homogenized after adding five volumes of homogenate buffer composed of $10 \mathrm{mM}$ HEPES buffer, $\mathrm{pH} 7.9,10 \mathrm{mM} \mathrm{KCl}, 0.1 \mathrm{mM}$ egtazic acid, $1 \mathrm{mM}$ dithiothreitol, and $0.5 \mathrm{mM}$ phenylmethylsulfonyl fluoride. The kidney homogenates were centrifuged at $3000 \mathrm{~g}$ for $15 \mathrm{~min}$ at $4^{\circ} \mathrm{C}$, and the supernatants were subsequently stored at $-80^{\circ} \mathrm{C}$. The protein extracts from the kidney tissues were separated on $12 \%$ SDS-PAGE gels and transferred to polyvinylidene difluoride membranes. Gapdh served as a loading control. Bmp7, Igf2, Tgf $\beta$, Hgf, and Ctgf expression were evaluated using rabbit polyclonal anti-Bmp7 (1:250; Bioworld Technology, USA), anti-Hgf (1:500; Abcom, USA), anti-Tgf $\beta$ (1:250; Bioworld Technology), anti-Ctgf (1:3000; Abcom), and anti-lgf2 (1:150; Bioworld Technology) antibodies, which were purified for immunogen affinity. The bands were quantified using the Image-Pro Plus software (USA).

\section{RNA extraction and complementary DNA (cDNA) synthesis}

Total RNA containing the miRNAs and cytokine mRNAs was extracted from kidney using Trizol reagent (Invitrogen, USA) according to the manufacturer's recommendations. Total RNA was eluted with RNase-free water and stored at $-80^{\circ} \mathrm{C}$, and RNA concentration was determined by spectrophotometry. Then, $1 \mu \mathrm{g}$ of RNA from each sample was reverse-transcribed into cDNA using random primers for the cytokine mRNAs, an miR483-specific stem-loop primer, an miR-483*-specific stem-loop primer, and a U6-specific stem-loop primer. The generated cDNAs were then subjected to conventional polymerase chain reaction (PCR). The stem-loop primer sequences of the miRNAs are shown in Table 1.

\section{Real-time PCR analyses}

Real-time PCR was performed using a 7500 Real-Time PCR System (Applied Biosystems, Germany) by the SYBER Green method, and primers specific to U6, Gapdh, miR-483, miR-483* and various cytokines (Bmp7, Igf2, Tgf $\beta$, Hgf, and Ctgf) that were synthesized by the Shengong Company (China). U6 and Gapdh genes were used as controls to normalize differences in the total RNA among the samples. Primer sequences are shown in Tables 2 and 3 . Variations in expression of miR-483 between different samples were calculated after normalization by $U 6$. 
Table 1. Stem-loop RT primers of miR-cDNA.

\begin{tabular}{ll}
\hline microRNA & \multicolumn{1}{c}{ Stem-loop RT primers } \\
\hline miR-483 & $5^{\prime}$-gtcgtatccagtgcgtgtcgtggagtcggcaattgcactggatacgacctccctt-3' \\
miR-483* & $5^{\prime}$-gtcgtatccagtgcgtgtcgtggagtcggcaattgcactggatacgacaagacgg-3' \\
\hline
\end{tabular}

Bmp7, Igf2, Tgf $\beta$, Hgf, and Ctgf were calculated and normalized by Gapdh. Results were normalized against the internal control using the $2-\Delta \Delta \mathrm{Ct}$ method, where $\mathrm{Ct}$ is defined as the fractional cycle number at which the fluorescence passes the fixed threshold.

\section{Target gene prediction}

The target genes of miR-483 and miR-483* were predicted using miRanda, PITA, and RNA hybrid software (12).

\section{Statistical analysis}

Data are reported as means \pm SD and were analyzed using SPSS 19.0 software (USA). To compare multiple group results, analysis of variance was used, followed by Dunnett's multiple comparison test. The correlation between miR-483 or miR-483* expression and the expression of various cytokines, namely $B m p 7$, Igf2, Tgf $\beta, H g f$, and Ctgf, in kidney from wild-type mice was examined using Pearson's correlation. Values of $\mathrm{P}<0.05$ were considered to be statistically significant.

\section{Results}

Formation of renal fibrosis in the UUO mouse model

Samples were scored according to the scoring system using Masson staining. Samples from the control group were scored as 0 , and the UUO samples obtained at 3 days and 1 and 2 weeks post-surgery were scored as $0-1$, 1 , and 3 , respectively. The kidney tubules dilated, and the extent of renal fibrosis worsened gradually. Furthermore, the kidney tubule walls became thin and atrophied, while the number of casts increased gradually (Figure 1).

Expression of and relationship among miR-483, miR-483* and the mRNAs for Bmp7, Igf2, Tgf, Hgf, and Ctgf

Real-time PCR was performed on the samples to evaluate the expression of miR-483, miR-483* and several cytokine mRNAs, including $B m p 7$, Igf2, Tgf $\beta$, $\mathrm{Hgf}$, and Ctgf. In addition, expression relationships among

Table 2. Primers for detecting miRNA expression by qRT-PCR.

\begin{tabular}{lc}
\hline microRNA & \multicolumn{1}{c}{ Forward primers } \\
\hline miR-483 & $5^{\prime}$-ggaagacgggagaagaga-3' \\
miR-483* & $5^{\prime}$ - attcactcctccctccc-3' \\
\hline
\end{tabular}

the above miRNAs and mRNAs were investigated in kidney tissue with varying extents of interstitial fibrosis. The expression of miR-483 and miR-483* was significantly higher at 1 and 2 weeks after surgery compared to the control group $(P<0.05)$, but was not significantly lower 3 days after surgery $(\mathrm{P}>0.05$; Figure $2 \mathrm{~A})$. Igf2 and $\mathrm{Hgf}$ mRNA expression increased significantly 1 and 2 weeks after surgery compared to the control group $(P<0.05)$, but was not significantly increased 3 days after surgery $(\mathrm{P}>0.05)$. Tgf $\beta$ and Ctgf mRNA expression increased significantly 3 days and 1 and 2 weeks after surgery compared to the control group $(\mathrm{P}<0.05)$, while $B m p 7$ mRNA expression decreased ( $P<0.05$; Figure $2 \mathrm{~B})$. Using Pearson correlation analysis, miR-483 expression positively correlated with $\operatorname{lgf2}, \operatorname{Tgf} \beta, \mathrm{Hgf}$, and Ctgf expression $(r=0.555,0.573,0.841,0.76$; Igf2 $\mathrm{P}<0.05$, others $\mathrm{P}<0.01)$ and negatively correlated with $B m p 7$ expression $(r=-0.72, \mathrm{P}<0.01$; Figure $2 \mathrm{C})$. In addition, miR-483* expression positively correlated with $\operatorname{lgf} 2, \operatorname{Tgf} \beta, \mathrm{Hgf}$, and Ctgf expression $(\mathrm{r}=0.805,0.623,0.792,0.874 ; \mathrm{P}<0.01)$ and negatively correlated with $B m p 7$ expression $(r=$ $-0.741, \mathrm{P}<0.01$; Figure 2D).

\section{Western blot analysis of abnormal cytokine expression in UUO mice}

Protein expression of $\operatorname{Tgf} \beta$ and Ctgf, both promoters of renal fibrosis, significantly increased 3 days and 1 and 2 weeks after surgery compared to the control group $(\mathrm{P}<0.01)$, while protein expression of $B m p 7$, an inhibitor of renal fibrosis, significantly decreased at 3 days and 1 and 2 weeks after surgery compared to the control group $(\mathrm{P}<0.01)$. Expression of $\mathrm{Hgf}$, an inhibitor of renal fibrosis,

Table 3. Primers for detecting kidney genes.

\begin{tabular}{llc}
\hline Gene name & \multicolumn{1}{c}{ Primers } & Tm $\left({ }^{\circ} \mathrm{C}\right)$ \\
\hline Bmp7 & F: aagacgccaaagaaccaaga & 59.9 \\
& R: cgcactctcctcacagtagta & \\
Tgf $\beta$ & F: aacaattcctggcgttacctt & 59.9 \\
& R: gaatcgaaagccctgtattcc & \\
Hgf & F: ccagaggtacgctacgaagtct & 60 \\
R: agtcttgcctgattctgtgtga & \\
Ftgf & $\begin{array}{l}\text { F: aactatgatgcgagccaactg } \\
\text { R: attggtaactcgggtggagat } \\
\text { Igf2 }\end{array}$ & 60.3 \\
& F: cgccttgtgctgcatcgctgctt & 60 \\
\hline
\end{tabular}

Tm: temperature. 


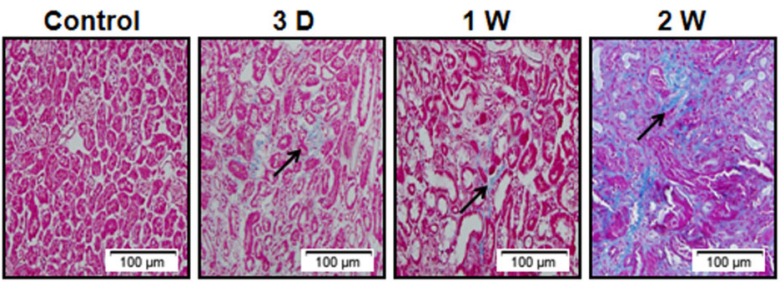

Figure 1. Masson staining of the kidney tissue. Samples were scored according to the scoring system using Masson staining: 0 , no fibrosis; 1 , mild fibrosis (10-30\% in area); 2 , moderate fibrosis ( $30-50 \%$ in area); 3 , severe fibrosis ( $>50 \%$ in area). Based on the average extent of histological change, the samples at 3 days post-surgery were scored $0-1$, at 1 week were scored 1 , and at 2 weeks were scored 3 . Arrows show fibrotic areas.

significantly increased 1 and 2 weeks after surgery compared to the control group $(\mathrm{P}<0.01)$. Expression of Igf2, the host gene for miR-483, significantly increased 3 days and 1 and 2 weeks after surgery compared to the control group ( $\mathrm{P}<0.01$; Figure 3$)$.

\section{Target genes}

PDGFb, TIMP2, and BMP1 are the target genes of miR-483. PDGFb, IGF2, CTGF, and TIMP2 are the target genes of miR-483* (Table 4). Indeed, there are hundreds of target genes of $\mathrm{miR}-483$ and $\mathrm{miR}-483^{*}$ as predicted using software. However, the above genes play functions in the metabolism of renal fibrosis or join in its pathogenesis. Thus, we chose those genes as target genes of miR483 or miR-483*.
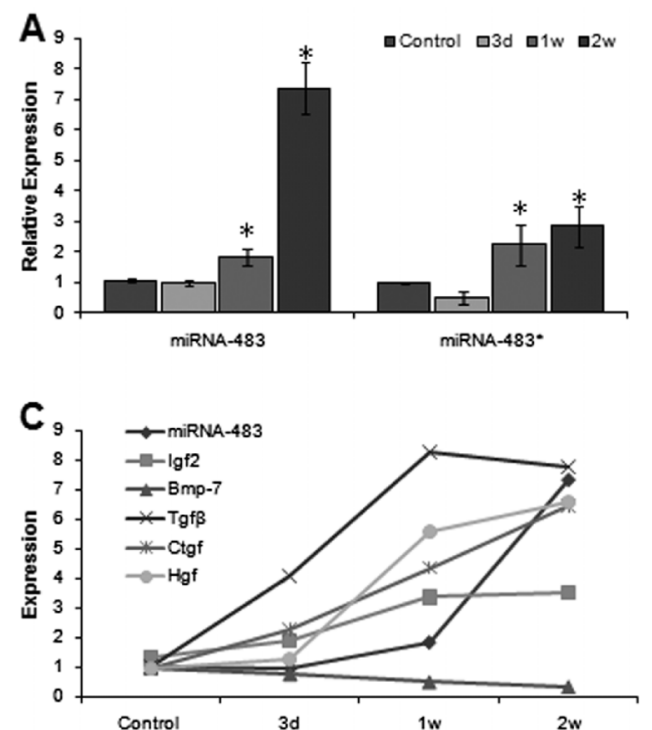

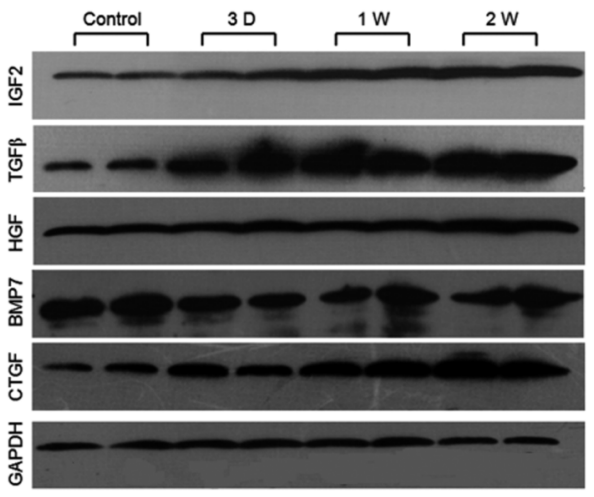

Figure 3. Western blot analysis of abnormal cytokine expression in mice after unilateral ureteral obstruction (UUO). TGF $\beta$ : transforming growth factor beta; HGF: hepatocyte growthpromoting factor; BMP7: bone morphogenetic protein-7; CTGF: connective tissue growth factor; GAPDH: glyceraldehyde 3phosphate dehydrogenase.

\section{Discussion}

Renal fibrosis is characterized by excessive deposition of ECM components in the kidney, which is the end result of an imbalance in the metabolism of the ECM molecule. "Amiss choices" in multiple pathways involved in renal tissue repair and inflammation can lead to the development of fibrosis. Many profibrotic mediators have been identified, such as TGF $\beta$. Unfortunately, the mechanism of renal fibrosis remains unclear. Recent studies have indicated that many miRNAs play crucial roles in the pathogenesis of
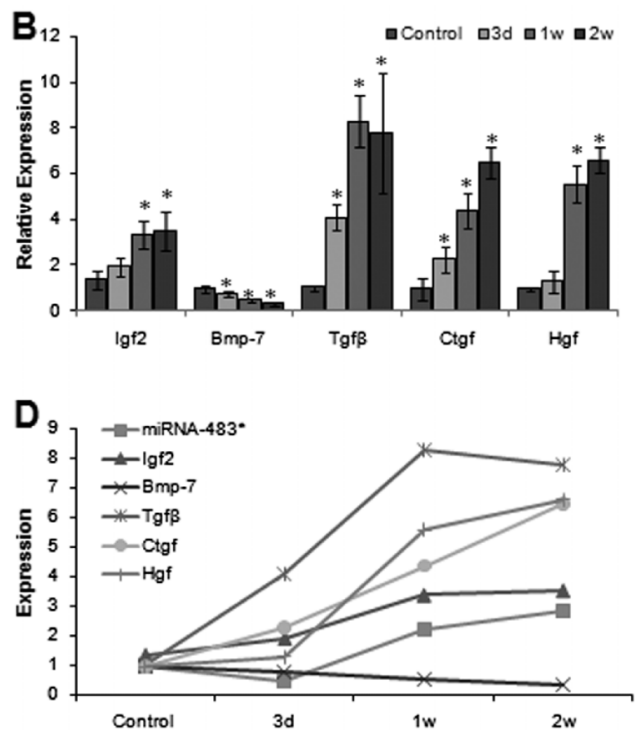

Figure 2. Expression of and relationships among miR-483, miR-483*, and the mRNAs for Bmp7, Igf2, Tgf $\beta$, Hgf and Ctgf. Real-time PCR was performed on the samples to evaluate the expression of miR-483, miR-483*, and several cytokine mRNAs, including Bmp7, Igf2, Tgf $\beta$, Hgf and Ctgf. d: days, w: week. ${ }^{*} \mathrm{P}<0.05$, compared to the control (Dunnett's multiple comparison test). 
Table 4. Target genes of miR-483 and miR-483*.

\begin{tabular}{|c|c|c|c|}
\hline microRNA & Target genes & & \\
\hline \multirow[t]{8}{*}{ miR-483 } & $P D G F b$ & 80 & $3^{\prime}$ gagggAAGAGAAGAGGGCAGAa 5' \\
\hline & & & $5^{\prime}$ uguugUUCCCCUCGUCCGUCUg 3' \\
\hline & $B M P 1$ & 287 & $3^{\prime}$ gagggaaGAGAAGAGGGCAGAa $5^{\prime}$ \\
\hline & & & $5^{\prime}$ cugcgggCUUCUCUGCCGUCUu 3' \\
\hline & TIMP2 & 1324 & $3^{\prime}$ gaGGGAAGAGAAGA-GG--GCAGAa 5' \\
\hline & & & 5' caCUCUUC-CUUCUGCCUGCGUCUu 3 \\
\hline & & 1992 & $3^{\prime}$ gaGGGAAG-AGAAGAGGGCAGAc 5' \\
\hline & & & $5^{\prime}$ ugCUUUUCCAGCCUCUCCCGUCUu 3' \\
\hline \multirow[t]{10}{*}{$\operatorname{miR}-483^{*}$} & $P D G F b$ & 56 & $3^{\prime}$ uucugcccuccccuCCUCACu $5^{\prime}$ \\
\hline & & & $5^{\prime}$ cccaugggguccuuGGAGUGa 3' \\
\hline & & 939 & $3^{\prime}$ uucugcccuccccuCCUCACu 5' \\
\hline & & & $5^{\prime}$ ccgaggcccucuguGGAGUGg 3' \\
\hline & IGF2 & 1374 & $3^{\prime}$ uucugccCUCCCCUCCUCACu 5' \\
\hline & & & 5' ucacacuGAUGUGGGGAGUGu 3' \\
\hline & CTGF & 334 & $3^{\prime}$ uucugcccucccuCCUCACu 5' \\
\hline & & & $5^{\prime}$ ugugccuacuuuuuGGAGUGu 3' \\
\hline & TIMP2 & 252 & $3^{\prime}$ uucugcccucCCCUCCUCACu 5' \\
\hline & & & 5' ccuugcuacaGGCAGGAGUGg 3' \\
\hline
\end{tabular}

renal fibrosis. For example, miR-21 amplifies TGF $\beta$ signaling and promotes fibrosis through a positive-feedback loop and other mechanisms (13-15). Additionally, miR-200 suppresses Zeb1, Zeb2, and TGF $\beta 2$ itself, which may inhibit TGF $\beta$-mediated EMT and prevent fibrosis (16). Moreover, TGF $\beta 1$-induced miR-192 expression in mesangial cells has been shown to increase collagen $1 \alpha 2$ expression and promote renal fibrosis (17).

Recent studies have reported the role of miR-483 in the pathogenesis of some diseases, such as adrenocortical tumors, asthma, and cardiovascular disease $(18,19)$. However, the function of miR-483 has not been reported in the pathogenesis of renal fibrosis. In our study, miR483 and miR-483* were highly expressed 1 and 2 weeks after surgery. Expression of miR-483 was highest 2 weeks after surgery and was higher than miR-483*; therefore, we indicate that miR-483 plays a role in renal fibrosis. In the maturation process of miRNAs, the miRNA* strand is degraded, although occasionally the miRNA* strand is also retained and functions as a mature miRNA (20). However, miR-483* expression increased but was lower than miR-483 expression; thus, we suspect that miR-483* acts as a partner for miR-483 in renal fibrosis, like miR-155* cooperating with its miRNA partner (miR-155) in the same physiological process (21). However, there is another possibility that miR-483* plays a converse role with miR-483.

miRNA miR-483 is located in the second intron of the Igf2 gene. Igf2 mRNA has been shown to be expressed in blastemal cells of the nephrogenic zone and in interstitial cells of the medullary region, and IGF2 has been shown to be involved in human renal development (7). IGF2 can activate the PI3-kinase/Akt pathway after binding to IGF1 receptor (22), and subsequent activation of this pathway can promote fibrosis. Ma et al. (10) reported that miR-483$5 \mathrm{P}$ is coexpressed with its host gene lgf2 and can be viewed as a functional partner for lgf2. In our study, we found that miR-483 and miR-483* expression positively correlated with lgf2, so we consider that they were coexpressed with Igf2 mRNA in the UUO model. Thus, we suspect that miR-483 may participate in renal fibrosis, through inhibiting the expression of some inhibitor mRNAs of renal fibrosis. Although lgf2 is the target gene of miR$483^{*}$, according to the results of target gene software prediction, it is presumed that miR-483* could inhibit expression of lgf2, in spite of coexpression between them. Thus, miR-483* has an adverse function with the host gene in renal fibrosis. However, in some tumors, miR-483-3P is highly expressed, but there is no concomitant increase in Igf2 (23). Thus, whether intronic miRNAs correlate with their host genes in terms of expression and function remains unclear. Further studies are needed regarding the role of miR-483 in the pathogenesis of renal fibrosis.

In the present study, we found that miR-483 and miR$483^{*}$ expression positively correlated with promoters ( $\operatorname{Tgf} \beta$, Ctgf $)$ and an inhibitor of renal fibrosis $(H g f)$, and negatively correlated with another inhibitor of renal fibrosis ( $B m p 7)$, as determined by statistical analysis. Interestingly, the extent of the correlations between miR-483 and miR-483* and the above cytokines is almost conformity. The results showed that miR-483 and miR-483* most likely both promote and inhibit renal fibrosis processes in direct or indirect ways. Furthermore, their overexpression enhanced one of the two processes for the promotion and inhibition of renal fibrosis. By predicting some target genes of miR-483 and miR-483*, we found that miR-483 and miR-483* 
participated in the pathogenesis of renal fibrosis via multitarget regulation. Interestingly, we found that $\mathrm{Hgf}$ mRNA and protein expression increased in our study, while some reports have indicated that $\mathrm{Hgf}$ expression decreased in the UUO model $(24,25)$. However, the Asanuma et al. (26) study indicated that $\mathrm{Hgf}$ mRNA expression increased in value for the UUO model, but the authors did not indicate the result. Thus, we think the expression pattern of $\mathrm{Hgf}$ needs to be studied.

Recent studies have revealed the role of some miRNAs in the pathogenesis of renal fibrosis, and miR-483 has been reported to play a role in some diseases $(18,23)$. However, the function of miRNA-483 in the pathogenesis of renal fibrosis remains unclear. Our study showed that expression of miR-483 and miR-483* was significantly altered in the renal fibrosis model. However, because the observation time was not long enough after surgery, we could not

\section{References}

1. Kopp JB. TGF-beta signaling and the renal tubular epithelial cell: too much, too little, and just right. J Am Soc Nephrol 2010; 21: 1241-1243, doi: 10.1681/ASN.2010060676.

2. Li JH, Zhu HJ, Huang XR, Lai KN, Johnson RJ, Lan HY. Smad7 inhibits fibrotic effect of TGF-Beta on renal tubular epithelial cells by blocking Smad2 activation. J Am Soc Nephrol 2002; 13: 1464-1472, doi: 10.1097/01.ASN. 0000014252.37680.E4.

3. Latronico MV, Condorelli G. MicroRNAs and cardiac pathology. Nat Rev Cardiol 2009; 6: 419-429, doi: 10.1038/nrcardio.2009.56.

4. Pandey AK, Agarwal P, Kaur K, Datta M. MicroRNAs in diabetes: tiny players in big disease. Cell Physiol Biochem 2009; 23: 221-232, doi: 10.1159/000218169.

5. Qi Y, Ma N, Yan F, Yu Z, Wu G, Qiao Y, et al. The expression of intronic miRNAs, miR-483 and miR-483*, and their host gene, Igf2, in murine osteoarthritis cartilage. Int $\mathrm{J}$ Biol Macromol 2013; 61: 43-49, doi: 10.1016/j.jibiomac. 2013.06.006.

6. Steck E, Boeuf S, Gabler J, Werth N, Schnatzer P, Diederichs $\mathrm{S}$, et al. Regulation of $\mathrm{H} 19$ and its encoded microRNA-675 in osteoarthritis and under anabolic and catabolic in vitro conditions. J Mol Med 2012; 90: 11851195, doi: 10.1007/s00109-012-0895-y.

7. Birnbacher R, Amann G, Breitschopf $H$, Lassmann $H$, Suchanek G, Heinz-Erian P. Cellular localization of insulinlike growth factor II mRNA in the human fetus and the placenta: detection with a digoxigenin-labeled cRNA probe and immunocytochemistry. Pediatr Res 1998; 43: 614-620, doi: 10.1203/00006450-199805000-00009.

8. Soon PS, Tacon LJ, Gill AJ, Bambach CP, Sywak MS, Campbell PR, et al. miR-195 and miR-483-5p identified as predictors of poor prognosis in adrenocortical cancer. Clin Cancer Res 2009; 15: 7684-7692, doi: 10.1158/10780432.CCR-09-1587.

9. Della Vittoria Scarpati G, Falcetta F, Carlomagno C, Ubezio $P$, Marchini S, De Stefano A, et al. A specific miRNA signature correlates with complete pathological response to neoadjuvant chemoradiotherapy in locally advanced rectal determine which changes were caused by expression of $\mathrm{miR}$ 483 and miR-483* during later stages of renal fibrosis, and this was a limitation of our study. Future studies are needed to find the real target gene of $\mathrm{miR}-483$ and $\mathrm{miR}-483^{*}$ regulating renal fibrosis from the predicted results, and the regulatory mechanisms. Several researchers have focused on therapeutic approaches that manipulate the expression of miRNAs connected with diseases (13). Further functional studies of miR-483 in the pathogenesis of renal fibrosis could offer a novel, reasonable approach to the treatment of renal fibrosis.

\section{Acknowledgments}

Research supported by Scientific Research Fund of Heilong jiang Provincial Education Department (\#12531321). cancer. Int J Radiat Oncol Biol Phys 2012; 83: 1113-1119, doi: 10.1016/j.jirobp.2011.09.030.

10. Ma N, Wang X, Qiao Y, Li F, Hui Y, Zou C, et al. Coexpression of an intronic microRNA and its host gene reveals a potential role for miR-483-5p as an IGF2 partner. Mol Cell Endocrinol 2011; 333: 96-101, doi: 10.1016/ j.mce.2010.11.027.

11. Kie JH, Kapturczak MH, Traylor A, Agarwal A, HillKapturczak N. Heme oxygenase-1 deficiency promotes epithelial-mesenchymal transition and renal fibrosis. $J \mathrm{Am}$ Soc Nephrol 2008; 19: 1681-1691, doi: 10.1681/ASN. 2007101099

12. Witkos TM, Koscianska E, Krzyzosiak WJ. Practical Aspects of microRNA Target Prediction. Curr Mol Med 2011; 11: 93-109, doi: 10.2174/156652411794859250.

13. Zhong X, Chung AC, Chen HY, Meng XM, Lan HY. Smad3mediated upregulation of miR-21 promotes renal fibrosis. J Am Soc Nephrol 2011; 22: 1668-1681, doi: 10.1681/ASN. 2010111168

14. Zarjou A, Yang S, Abraham E, Agarwal A, Liu G. Identification of a microRNA signature in renal fibrosis: role of miR-21. Am J Physiol Renal Physiol 2011; 301: F793F801, doi: 10.1152/ajprenal.00273.2011.

15. Thum T, Gross C, Fiedler J, Fischer T, Kissler S, Bussen M, et al. MicroRNA-21 contributes to myocardial disease by stimulating MAP kinase signalling in fibroblasts. Nature 2008; 456: 980-984, doi: 10.1038/nature07511.

16. Gregory PA, Bracken CP, Smith E, Bert AG, Wright JA, Roslan S, et al. An autocrine TGF-beta/ZEB/miR-200 signaling network regulates establishment and maintenance of epithelial-mesenchymal transition. Mol Biol Cell 2011; 22 1686-1698, doi: 10.1091/mbc.E11-02-0103.

17. Kato M, Zhang J, Wang M, Lanting L, Yuan H, Rossi JJ, et al. MicroRNA-192 in diabetic kidney glomeruli and its function in TGF-beta-induced collagen expression via inhibition of E-box repressors. Proc Natl Acad Sci U S A 2007; 104: 3432-3437, doi: 10.1073/pnas.0611192104.

18. Patterson EE, Holloway AK, Weng J, Fojo T, Kebebew E. MicroRNA profiling of adrenocortical tumors reveals miR-483 
as a marker of malignancy. Cancer 2011; 117: 1630-1639, doi: $10.1002 /$ cncr.25724.

19. Garbacki N, Di Valentin E, Huynh-Thu VA, Geurts P, Irrthum A, Crahay $\mathrm{C}$, et al. MicroRNAs profiling in murine models of acute and chronic asthma: a relationship with mRNAs targets. PLoS One 2011; 6: e16509, doi: 10.1371/journal.pone.0016509.

20. Patel V, Noureddine L. MicroRNAs and fibrosis. Curr Opin Nephrol Hypertens 2012; 21: 410-416, doi: 10.1097/ MNH.0b013e328354e559.

21. Zhou $H$, Huang $X$, Cui $H$, Luo $X$, Tang $Y$, Chen $S$, et al. miR155 and its star-form partner miR-155* cooperatively regulate type I interferon production by human plasmacytoid dendritic cells. Blood 2010; 116: 5885-5894, doi: 10.1182/ blood-2010-04-280156.

22. Chao W, D'Amore PA. IGF2: epigenetic regulation and role in development and disease. Cytokine Growth Factor Rev 2008; 19: 111-120, doi: 10.1016/j.cytogfr.2008.01.005.
23. Veronese A, Lupini L, Consiglio J, Visone R, Ferracin M, Fornari $F$, et al. Oncogenic role of miR-483-3p at the IGF2/ 483 locus. Cancer Res 2010; 70: 3140-3149, doi: 10.1158/ 0008-5472.CAN-09-4456.

24. Mizuno S, Matsumoto K, Nakamura T. Hepatocyte growth factor suppresses interstitial fibrosis in a mouse model of obstructive nephropathy. Kidney Int 2001; 59: 1304-1314, doi: 10.1046/j.1523-1755.2001.0590041304.x.

25. Klahr S, Morrissey J. Obstructive nephropathy and renal fibrosis: The role of bone morphogenic protein-7 and hepatocyte growth factor. Kidney Int Suppl 2003; S105S112, doi: 10.1046/j.1523-1755.64.s87.16.x.

26. Asanuma $\mathrm{H}$, VanderBrink $\mathrm{BA}$, Campbell $\mathrm{MT}$, Hile $\mathrm{KL}$, Zhang $H$, Meldrum DR, et al. Arterially delivered mesenchymal stem cells prevent obstruction-induced renal fibrosis. J Surg Res 2011; 168: e51-e59, doi: 10.1016/j.jss. 2010.06.022. 